\title{
Integrated Silicon-on-Insulator Spectrometer with Single Pixel Readout for Mid-Infrared Spectroscopy
}

\author{
Anton Vasiliev, Student Member, IEEE, Muhammad Muneeb, Jeroen Allaert, \\ Joris Van Campenhout Member, IEEE, Roel Baets, Fellow, IEEE, and Günther Roelkens, Senior Member, IEEE
}

\begin{abstract}
Mid-infrared spectroscopy in the 2-4 $\mu \mathrm{m}$ wavelength range is of cardinal value for many sensing applications. Current solutions involve bulky and expensive systems to operate. Silicon-on-Insulator (SOI) waveguide technology offers means to miniaturize the different parts of the spectrometer. However, the development of on-chip detectors for the mid-infrared wavelength range is in its infancy and the characteristics are not on par with their discrete (cooled) counterparts. In this work, a compact and cheap mid-infrared spectrometer is realized by integrating a SOI Arrayed Waveguide Grating (AWG) spectrometer operating in the $2.3 \mu \mathrm{m}$ wavelength range with a high performance midinfrared photodiode. The AWG has twelve output channels with a spacing of $225 \mathrm{GHz}(4 \mathrm{~nm})$ and a free spectral range (FSR) of $3150 \mathrm{GHz}(56 \mathrm{~nm})$, which are simultaneously collected by a single, transistor outline (TO)-packaged extended InGaAs PIN photodiode. The response of each AWG channel is discerned by time-sequentially modulating the optical power in each output channel using integrated Mach-Zehnder based (MZI) thermooptic modulators with a $\pi$-phase shift power consumption of $\approx \mathbf{5 0}$ mW. As an example, the absorption spectrum of a $0.5 \mathrm{~mm}$ thick polydimethylsiloxane sheet (PDMS) is sampled and compared to a benchtop spectrometer to good agreement.
\end{abstract}

Index Terms-infrared spectroscopy, integrated optoelectronics, optical spectroscopy, silicon on insulator technology, spectroscopy

\section{INTRODUCTION}

$\mathbf{S}$ ILICON photonics has been tremendously successful in the area of optical communication [1]-[4]. The main reason is that the Silicon-on-Insulator (SOI) platform can benefit from well-established silicon electronics processes to manufacture photonic devices in high volumes and high yield but at a low cost [5]. More over, the high refractive index contrast of SOI optical waveguides allows for a very tight bend radius and therefore, ultra compact devices can be fabricated. The portfolio of SOI waveguide technology applications has expanded towards applications such as biosensing [6]-[8], biomedical analysis [9], [10], structural analysis [11], [12] and gas sensing [13], [14]. Over the years, there has been progressively more interest towards longer wavelengths for a variety of new sensing markets

Manuscript received XX XX, 2018; revised August XX, XX.

A. Vasiliev, M. Muneeb, J. Allaert, R. Baets and G. Roelkens are with the Photonics Research Group, Department of Information Technology, imec, and the Center for Nano- and Biophotonics, Ghent University, TechnologieparkZwijnaarde 15, B-9052 Ghent, Belgium (e-mail: Anton.Vasiliev@UGent.be; Muhammad.Muneeb@UGent.be; Roel.Baets@UGent.be; Gunther.Roelkens@UGent.be).

J. Van Campenhout is with IMEC, Kapeldreef 75, B-3001 Leuven, Belgium (e-mail: Joris.VanCampenhout@imec.be).

A. Vasiliev thanks Research Foundation - Flanders (FWO) for a $\mathrm{PhD}$ fellowship. and in particular for mid-infrared absorption spectroscopy. It is well-known that gas molecules have strong rovibrational resonances in the mid-infrared $(>2 \mu \mathrm{m})$ wavelength range [15]. It is possible to access these longer wavelengths (2-4 $\mu \mathrm{m})$ in the same SOI waveguide platform without leaving the well-established CMOS fabrication technology. However, this transition requires careful redesign of all the different subcomponents at these wavelengths [16]-[19]. The realization of silicon photonics components in this wavelength range could enable economical and compact optical sensors with superior performance in sensitivity, power consumption and portability compared to current bulky solutions. The absorption of $\mathrm{SiO}_{2}$ beyond $4 \mu \mathrm{m}$ wavelength and the substrate leakage set the upper wavelength limit for traditional SOI waveguide technology [16], [20], [21].

There are several approaches to realize a compact and cheap spectroscopic system and the required wavelength coverage and resolution determine the best solution. For spectroscopy of gases, a high resolution spectrum in a narrow wavelength range is typically required. This can be realized with midinfrared distributed feedback (DFB) laser sources which can be thermally tuned over a narrow wavelength range [22][24]. The wavelength range can be further increased by fabricating an array of DFB lasers with slightly different central wavelengths. The central wavelength of each DFB laser can be adjusted by small alterations in the design [25]. The output from such a laser array could be multiplexed to a single diffraction limited output beam with low loss by using a highperformance SOI wavelength (de)multiplexer (WDM) such as an Arrayed Waveguide Grating (AWG) or Planar Concave Grating (PCG) [26], [27].

For spectroscopy applications involving broad absorption features such as liquids and solids where wavelength coverage is more important than resolution, a more costefficient solution including a broadband light source and a dispersive element is more suitable. Substantial effort is devoted to develop such novel spectrometers that are compact and can be integrated with photonic ICs. Some examples include spatial heterodyne spectrometers (SHS) [28], on-chip microelectromechanical (MEMS)-based Fourier Transform Infared Spectrometers (FTIR) [29], stationary FTIRs [30] and AWGs [31]. The AWG is a versatile component for spectroscopy which can easily be designed with good performance. The free spectral range (FSR) and channel spacing can be tailored to fit the application [32].

It is possible to heteregeneously integrate III-V-on-Silicon detectors on the photonic IC with the spectrometer in the 2-4 


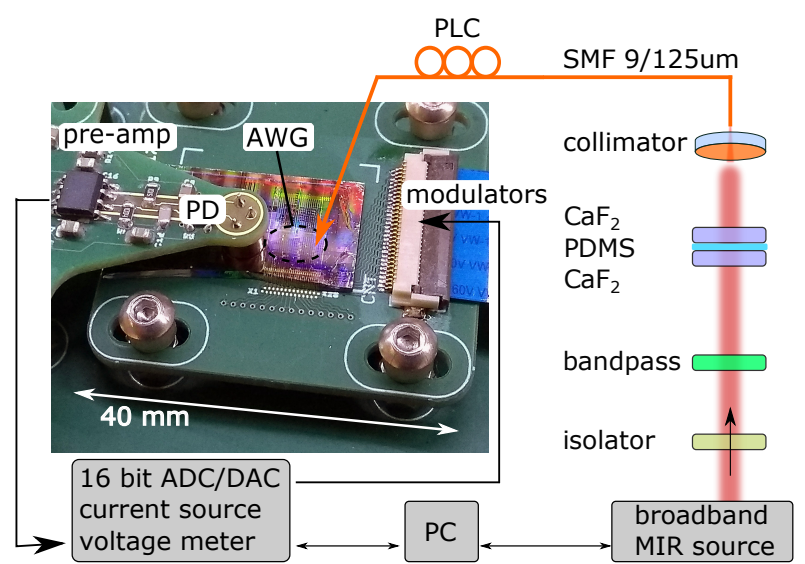

(a)

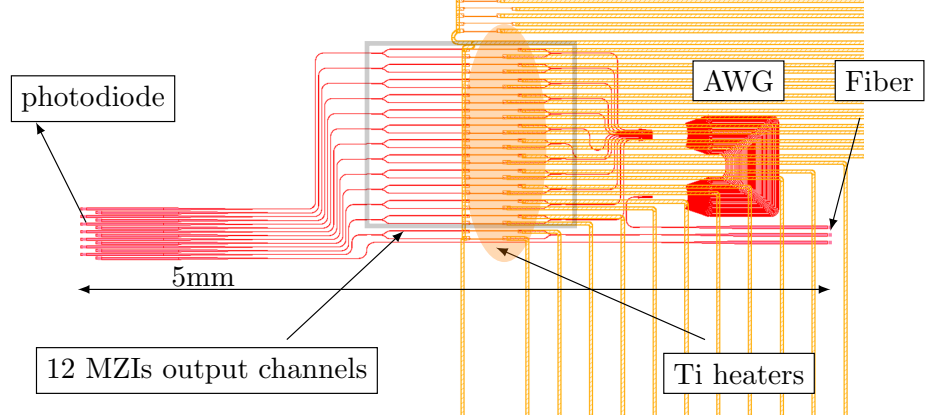

(b)

Fig. 1. Schematic of the spectroscopy setup using a SOI AWG spectrometer with a single external detector (a). A $\mathrm{Cr}^{2+}: \mathrm{ZnS}$ laser working in ASE mode with a bandpass filter is used as the broadband mid-infrared source to determine the absorption spectrum of a $0.5 \mathrm{~mm}$ thick PDMS sheet, held between two $\mathrm{CaF}_{2}$ windows. The light is coupled to a standard 9/125 fiber pigtail. The TO-can with the photodiode is positioned directly on top of the output grating couplers of the chip and collects all twelve output channels of the AWG simultaneously. Design layout of the AWG (b). The output channels are modulated time-sequentially using (balanced) MZI thermo-optic modulators. A $\pi$-phase shift in each channel is achieved by dissipating $\approx 50 \mathrm{~mW}$ in the metal (Ti/Au) resistors placed $0.9 \mu \mathrm{m}$ above the optical waveguide. The bandpass filter, PDMS and PD are not used in the passive characterization of the grating coupler efficiency and AWG response. Instead, a second single mode fiber is used to collect the output light and a mid-IR OSA is used as a sensitive power- and wavelength meter.

$\mu \mathrm{m}$ wavelength range [31], [33]. However, existing packaged (cooled) mid-infrared photodetectors currently outperform the on-chip solutions. We present an approach for integrating a single standard TO-can packaged InGaAs PIN photodiode with a twelve channel AWG with $225 \mathrm{GHz}(4 \mathrm{~nm})$ channel spacing. In order to distinguish the different channels, the output arms of the AWG are modulated using on-chip balanced MachZehnder interferometers (MZIs) by thermal tuning [34]. To showcase the mini-spectrometer, the absorption spectrum of polydimethylsiloxane (PDMS) in the $2.3 \mu \mathrm{m}$ wavelength range is recovered.

\section{FABRICATION}

The AWG is fabricated on a $200 \mathrm{~mm}$ SOI wafer with a 400 $\mathrm{nm}$ thick crystalline $\mathrm{Si}$ device layer and $2 \mu \mathrm{m}$ buried oxide layer thickness. Rib waveguides and grating couplers (GCs) are defined with a $180 \mathrm{~nm}$ deep etch and are cladded with $\mathrm{SiO}_{2}$ and planarized down to the silicon device layer. A thin $0.9 \mu \mathrm{m}$ layer of $\mathrm{SiO}_{x}$ is deposited on top. 100/10 nm thick $\mathrm{Ti} / \mathrm{Au}$ resistors are defined on the arms of the modulators. The resistors measure $200 \times 2 \mu \mathrm{m}^{2}$ and realize a $\pi$-phase shift with $\approx 50 \mathrm{~mW}$ of power dissipation. As a final step, the chip is passivated with a benzocyclobutene (BCB) polymer layer. The circuit is designed for $2.3 \mu \mathrm{m}$ wavelength and TE polarized light. The AWG has twelve channels with $225 \mathrm{GHz}$ $(4 \mathrm{~nm})$ channel spacing and an FSR of $3150 \mathrm{GHz}(56 \mathrm{~nm})$. The MZI modulators of the photonic chip are wire bonded to a printed circuit board (PCB) and are addressed by a home-built 16-bit current source through USB. An uncooled Hamamatsu G12183-010K PIN photodiode (PD) is fixed to a pre-amplifier PCB with a variable gain up to $10^{6} \mathrm{~V} / \mathrm{A}$. The electronics to drive the modulators and read the photodiode response are based on off-the-shelf components. The PD is manually positioned and fixed $0.5 \mathrm{~mm}$ above the output grating coupler array of the AWG, see Fig. 1(a). The output grating array covers an area of $300 \times 140 \mu \mathrm{m}^{2}$ and all the channels are simultaneously collected by the photosensitive area of the PD, $1 \mathrm{~mm}$ in diameter. The PD response is read-out using the aforementioned 16-bit ADC. A microcontroller is programmed to send the read/write commands in blocks with a resulting PD sampling rate of approximately $24 \mathrm{kSa} / \mathrm{s}$. A $0.5 \mathrm{~mm}$ thick PDMS foil was prepared using the Sylgard 184 (10:1) elastomer kit and a suitable mold.

\section{AWG CHARACTERIZATION}

The performance of the photonic circuit is characterized using a Continuous Wave (CW) single mode $\mathrm{Cr}^{2+}: \mathrm{ZnS}$ solid state laser from IPG Photonics. The free space output power from the laser is coupled to a standard $9 / 125$ single mode fiber as in Fig. 1(a) but without the bandpass filter, PDMS and PD. The power in the fiber is between one and few tens of $\mathrm{mW}$ depending on the laser wavelength. A second single mode fiber collects the output light from the IC and a Yokogawa AQ6375 Optical Spectrum Analyzer (OSA) was used as a sensitive detector and wavelength meter. A cooled extended InGaAs PDA10DT photodetector from Thorlabs was used as a reference, connected to a 1/99 fiber splitter to compensate for slow time-fluctuations of the laser power. The AWG response with the MZI modulator arms is normalized to a reference waveguide and the results are shown in Fig. 2(a). The channel spacing is $225 \mathrm{GHz}(4 \mathrm{~nm})$ and one FSR spans $3150 \mathrm{GHz}(56 \mathrm{~nm})$. The insertion loss is around $2 \mathrm{~dB}$ and the crosstalk between channels is $20 \mathrm{~dB}$. The grating coupler efficiency is calculated to be around $-9 \mathrm{~dB} / \mathrm{GC}$ at $2340 \mathrm{~nm}$ peak wavelength with 18 degrees fiber coupling angle, see Fig. 2(b). By switching the output fiber with the single pixel $\mathrm{PD}$, the combined response of the AWG channels is retrieved. The PD voltage is normalized to the power in the fiber towards 


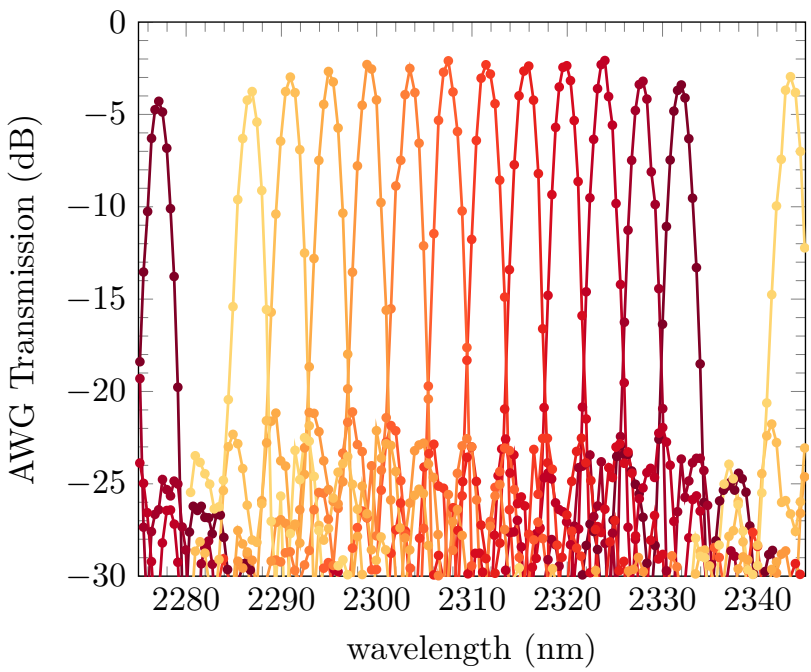

(a)

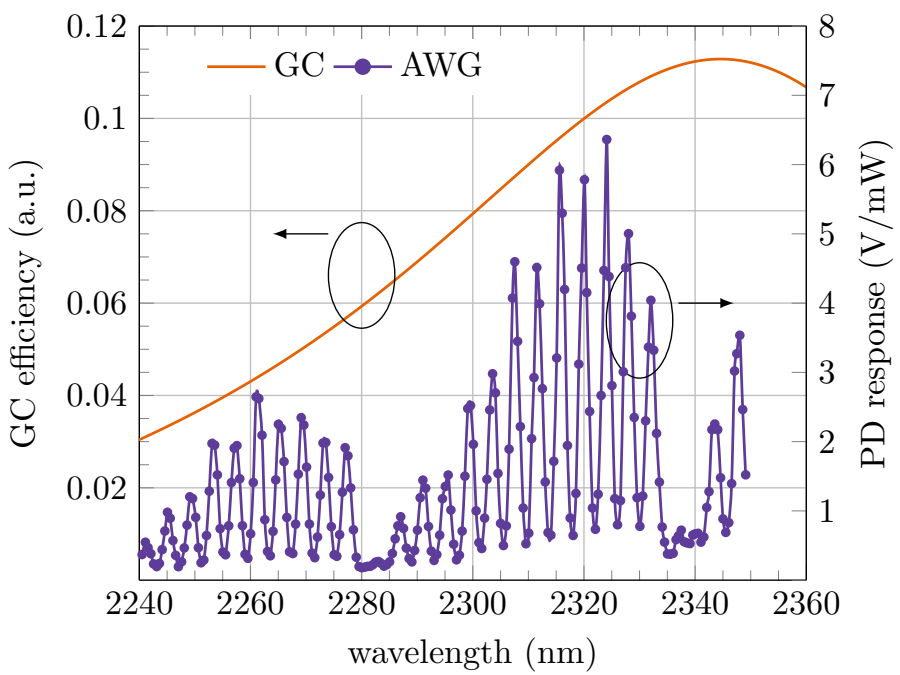

(b)

Fig. 2. The AWG channels from layout Fig. 1(b) are characterized using a single-mode tunable CW laser and normalized to a reference waveguide (a). The channel spacing is $225 \mathrm{GHz}(4 \mathrm{~nm})$ and one FSR spans $3150 \mathrm{GHz}(56 \mathrm{~nm})$. The insertion loss is around $2 \mathrm{~dB}$ and the crosstalk between channels is $20 \mathrm{~dB}$. The output light is collected with a single mode fiber and the power and wavelength are obtained with the Yokogawa OSA. The grating coupler efficiency is estimated from the reference waveguide measurement (b). The insertion loss is $9 \mathrm{~dB}$ at $2340 \mathrm{~nm}$ peak wavelength and 18 degrees fiber coupling angle. The AWG response, normalized to the optical power in the fiber, is obtained from all channels simultaneously by using the TO-can PD as the detector. The PD response follows the GC efficiency curve and all twelve channels are visible. An additional envelope is observed due to limited dimensions and exact position of the PD on top of the output GC array. The beams coming from the edges of the output array are captured less efficiently. The position of the PD is fixed with screws but can be readjusted if needed.

the chip, calculated using the laser source reference detector. All twelve channels are clearly distinguishable. The combined AWG response follows the grating coupler trend with an additional envelope. The relative position of the output GC array to the PD and the finite aperture of the PD determine this envelope, see also Fig. 1. When the PD is positioned in the center of the GC array, the outer channels are captured less efficiently. The position of the PD is fixed with M4 screws and can be readjusted along $\mathrm{x}$ - and $\mathrm{y}$-slits.

The MZI modulators are characterized using the same method as above but now the wavelength is fixed to the peak transmission of the AWG channel and electrical power is dissipated through the resistor on one of the arms of the MZI. The increased local temperature of the Si waveguide induces a change in effective index through the thermooptic effect. The applied voltage and power are set with a Keithley 2400 measurement unit, see Fig. 3(a). The resistance is approximately $1200 \Omega$ for all heaters and the extinction ratio is more than $30 \mathrm{~dB}$. A $\pi$-phase shift is obtained when $P_{\pi}=49$ $\mathrm{mW}$ power is dissipated. In the spectroscopy experiment where all twelve channels are collected simultaneously by the PD, the dissipated heat $P_{\pi}$ on one channel causes a drop of $1.5 / 1.68 \approx 11 / 12$ of the amplified PD response as expected, see inset in Fig. 3(a). Due to small variations in fabrication, $P_{\pi}$ varies between $48-56 \mathrm{~mW}$ and the corresponding $V_{\pi}$ between 6-7 $\mathrm{V}$ for the 12 channels of the AWG. The AWG channel transmission is shown when the heater is switched on $V=V_{\pi}$ and when it is off $V=0$, see Fig. 3(b). The pitch between the MZI channels is $100 \mu \mathrm{m}$. No thermal cross-talk between adjacent channels was observed and therefore must be lower than the optical crosstalk of the AWG, see Fig.2(a).

\section{AbSORption Spectroscopy}

To showcase the single pixel mini-spectrometer, the absorption spectrum of PDMS in the $2.3 \mu \mathrm{m}$ wavelength region is probed using three different methods. First, the absorption spectrum is determined using the Agilent 680 FTIR spectrometer in transmission, see Fig. 4(a). The importance of the 2-3 $\mu \mathrm{m}$ wavelength range is indeed also valid in this case as can be seen from the increased absorption cross section as compared to the $1.5-2 \mu \mathrm{m}$ wavelength range.

Secondly, the PDMS sheet is placed between two $4 \mathrm{~mm}$ thick $\mathrm{CaF}_{2}$ windows in the beam path of the $\mathrm{Cr}^{2+}: \mathrm{ZnS}$ laser operating in amplified spontaneous emission (ASE) mode. The fiber coupled power is measured by the mid-infrared Yokogawa OSA with $0.1 \mathrm{~nm}$ resolution both with (PDMS), and without (Ref) the PDMS sample. The measured total optical power is $11.5 \mu \mathrm{W}$ and $31.4 \mu \mathrm{W}$ respectively. When PDMS is introduced in the beam path, the collimator has to be realigned to a new optimum. The spectrum shows spectral ripple due to residual thin film interference effects of the bandpass filter. The normalized FTIR transmission of the PDMS sample and the bandpass filter is also shown in Fig. 4(b).

Lastly, the AWG is used as a dispersive spectrometer to recover the response of PDMS at the AWG peak wavelengths, marked as black crosses on the top axis in Fig. 4(b). Ideally, the AWG response would be limited to within one FSR closest to the PDMS absorption feature. The available bandpass filter is centered at $2305 \mathrm{~nm}$ with a Full Width at Half Maximum (FWHM) of $50 \mathrm{~nm}$ and overlaps unintentionally with the next FSR on the blue side of the PDMS absorption peak. As a result, the longer wavelength channels of the AWG spectrometer will show additional crosstalk. 


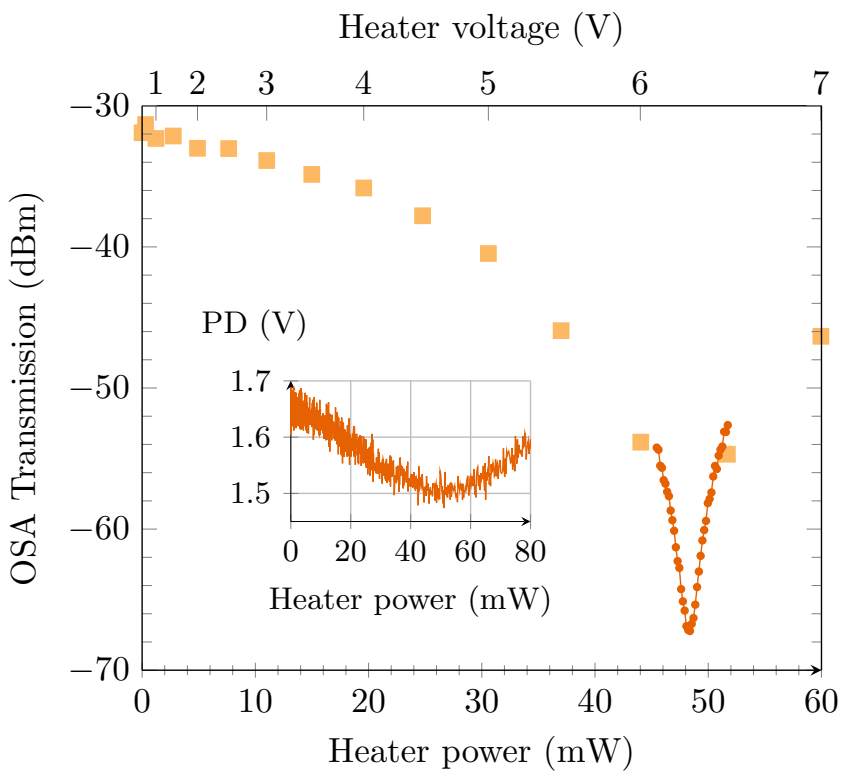

(a)

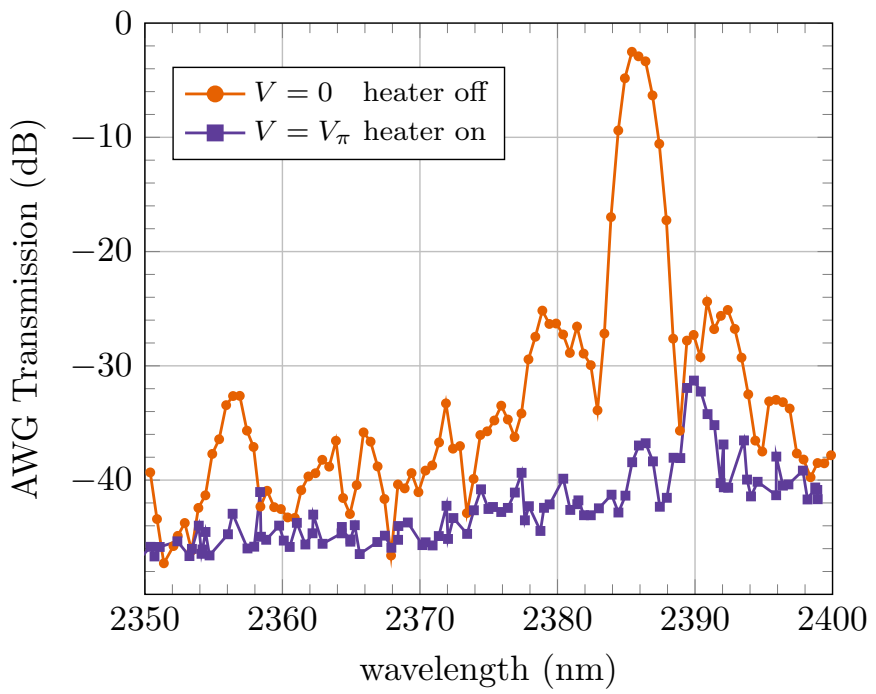

(b)

Fig. 3. The characterization of a MZI modulator as a function of dissipated power is shown for one channel of the AWG (a). A coarse and a fine voltage sweep is performed to find the minimum transmission of the channel at $2386 \mathrm{~nm}$ wavelength. The inset shows the amplified PD response as a function of dissipated heat on one channel when all twelve channels are collected simultaneously by the single pixel for the setup as in Fig. 1. The resistance is approximately 1200 $\Omega$ for all heaters and the extinction ratio of the MZI is more than $30 \mathrm{~dB}$. A $\pi$-phase shift is obtained when $P_{\pi}=49 \mathrm{~mW}$ power is dissipated. Due to small variations in fabrication, $P_{\pi}$ varies between $48-55 \mathrm{~mW}$ and the corresponding $V_{\pi}$ between 6-7 V for the 12 channels of the AWG. The channel transmission is shown when the heater is switched on $\left(V=V_{\pi}\right)$ and when it is off $(V=0)(\mathrm{b})$.

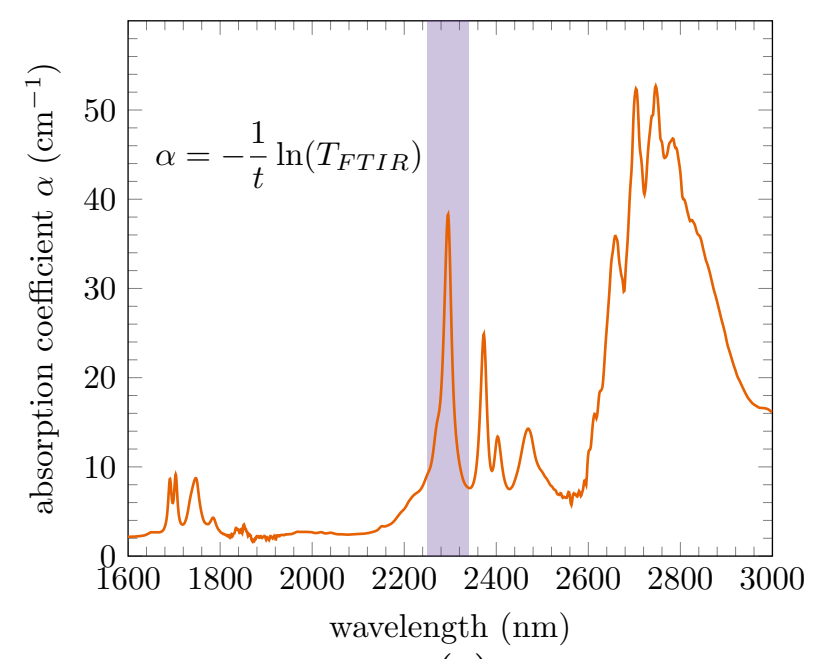

(a)

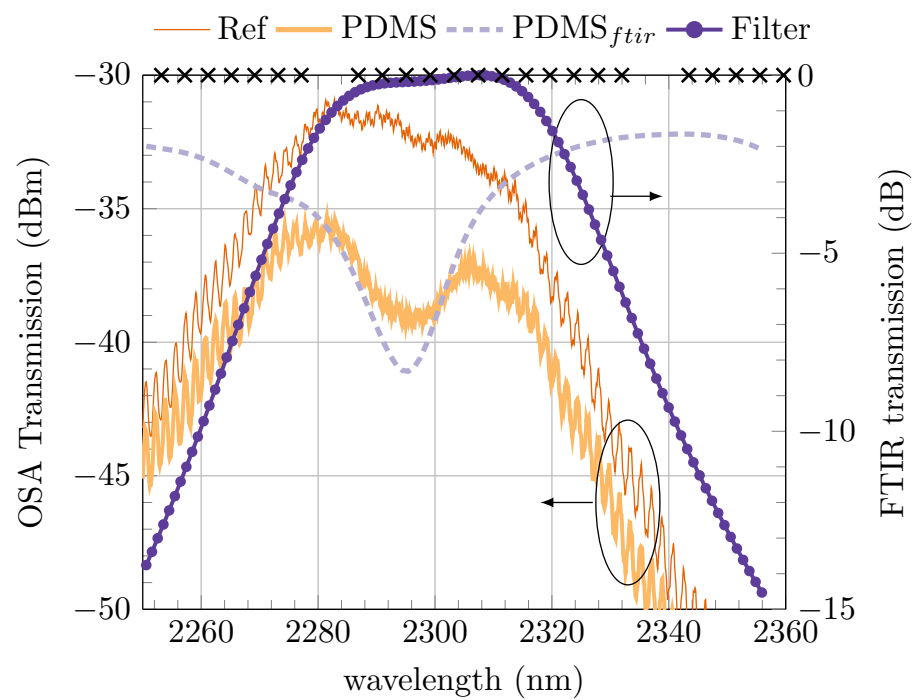

(b)

Fig. 4. The absorption coefficient of PDMS in the near- to mid-infrared wavelength region is estimated by using the Lambert-Beer law and the FTIR transmission spectrum of a $(t=0.5 \mathrm{~mm})$ thick PDMS foil (a). The wavelength region for the absorption spectroscopy experiment is colored. After passing the bandpass filter, see Fig. 1(a), the optical spectrum coupled into the fiber is measured using the Yokogawa OSA: in the case when PDMS is present (PDMS) and when it is removed (Ref) (b). The measured total optical power is $11.5 \mu \mathrm{W}$ and $31.4 \mu \mathrm{W}$ respectively. The normalized FTIR transmission of the PDMS sample $\left(\mathrm{PDMS}_{\text {ftir }}\right.$ ) and the bandpass (Filter) is also shown. The black crosses on the top axis show the AWG peak wavelengths. Ideally, the AWG response would be limited to within one FSR closest to the PDMS absorption feature. The filter is centered at $2305 \mathrm{~nm}$ with a FWHM of $50 \mathrm{~nm}$ and overlaps unintentionally with the next FSR on the blue side of the PDMS absorption peak. As a result, the longer wavelength channels of the AWG spectrometer will show additional crosstalk.

The combined output from the channels of the AWG results in a PD signal of $3.7 \mathrm{~V}$ for the reference measurement and $1.4 \mathrm{~V}$ when PDMS is introduced. The optical power inside each channel can be recovered by modulating each channel time-sequentially [34]. The modulation depth of the heaters is chosen such that it corresponds closely to a $\pi$-phase shift of each MZI. Slight differences in fabrication lead to drive voltages $V_{\pi}$ that are spread between 6 and $7 \mathrm{~V}$ and the 
(a)

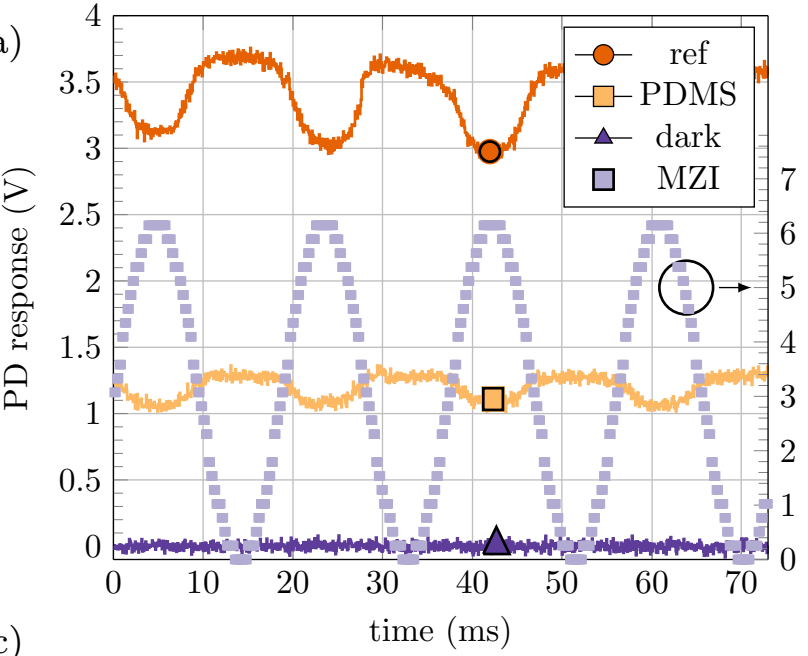

(c)

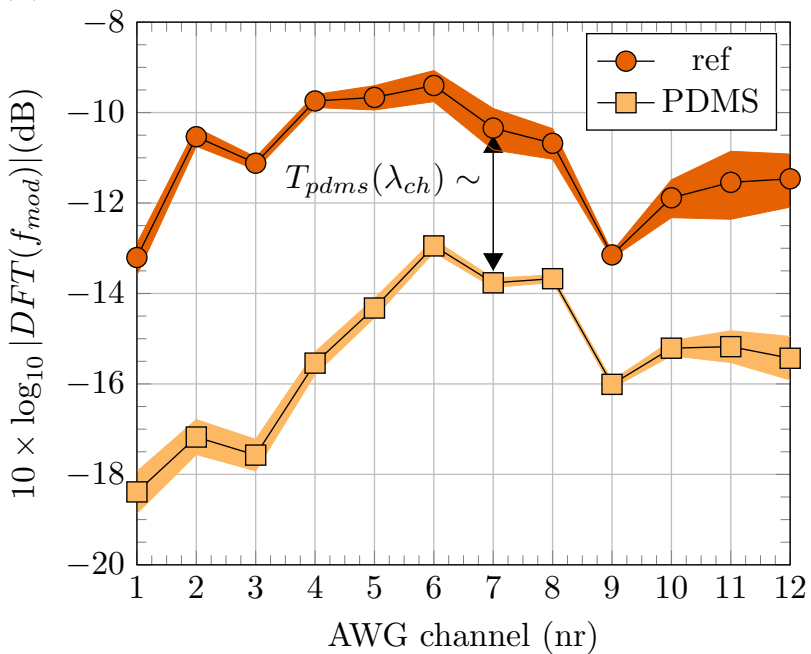

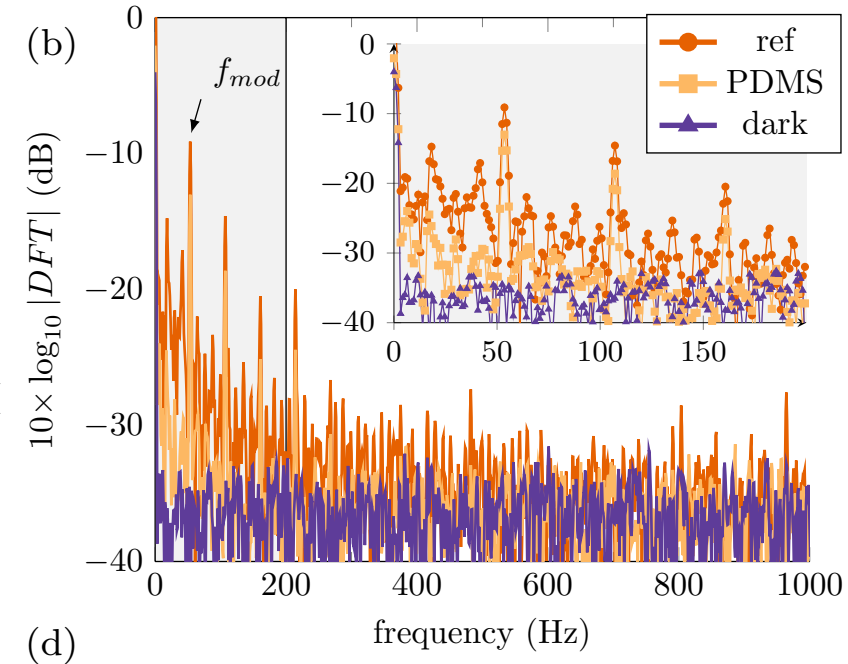

$(\mathrm{d})$

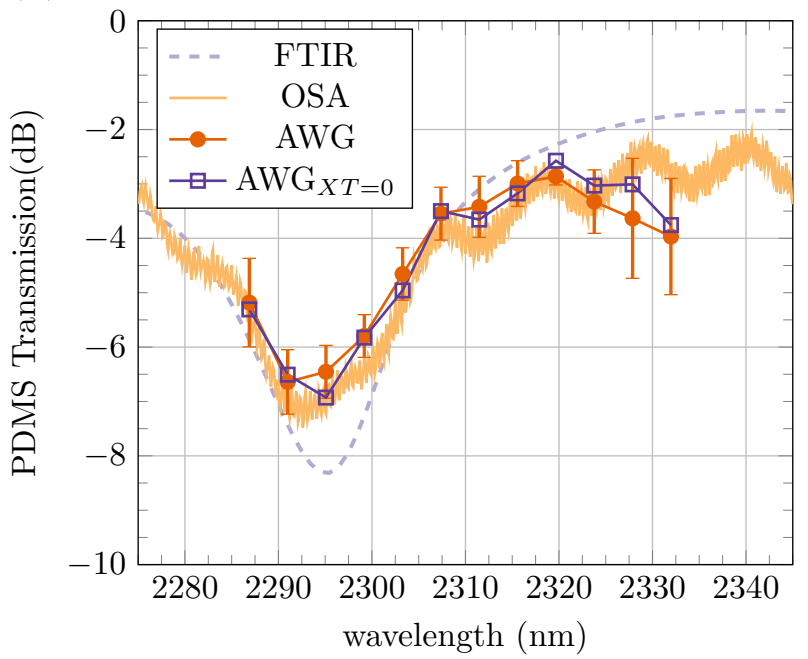

Fig. 5. Response of the photodiode to a sinusoidal modulation of one AWG channel. The first thousand sample points are shown for three cases: prior to the chip, the light passes through a $0.5 \mathrm{~mm}$ thick PDMS sheet, a reference measurement without PDMS and a dark measurement without the light source (a). The sample points are collected by a microcontroller in packets and are not linked to an external clock. The resulting PD sampling rate is $\approx 24 \mathrm{kSa} / \mathrm{s}$. The time intervals between samples can be slightly different and the given time axis is an appproximation. For each setpoint of the heater, four consecutive PD readings are collected. The logarithmic DFT spectrum of the PD response is shown (b). The difference in maxima between the 'PDMS' and 'ref' measurement at the modulation frequency $f_{m o d}$ is used to determine the spectral response of PDMS at the peak wavelength of each AWG channel (c). The colored region boundaries are the $\mu \pm \sigma$ values for seven different measurements. The optical modulation depth difference of the PD between the PDMS and reference measurement is plotted for each AWG channel with the corresponding channel peak wavelength (d). The results agree well with the results obtained by measuring the PDMS transmission using the benchmark mid-IR Yokogawa OSA. Main cause of variation is power and alignment drift of the source to the spectrometer. Taking the OSA spectrum as the true response of PDMS in this setup, calculations show that an idealized AWG with zero crosstalk XT would produce almost identical results.

corresponding switching power varies between $48-56 \mathrm{~mW}$. An example of modulation of one such channel is shown in Fig. 5(a). For each heater bias point, four consecutive PD value are collected and averaged. The PD output is collected for 1 second for each channel consecutively with a sampling rate of $24 \mathrm{kSa} / \mathrm{s}$. This corresponds to the response to 50 sinusoidal heating cycles for each channel. After sweeping the twelve channnels of the AWG, the discrete Fourier Transform (DFT) of the signal is performed on the four-piece averaged output using the fast-fourier transform (FFT) algorithm with a Kaiser smoothing window. The result for one channel is shown in Fig. 5(b). The absolute value of the DFT spectrum at the modulation frequency is proportional to the optical power modulation of that channel. This value is obtained for each of the twelve channels for the case when there is no PDMS in the free space beam path and when it is introduced (ref), see Fig. 5(c). The difference between the two is a measure for the absorption spectrum of PDMS, sampled at the peak wavelengths of the AWG channels. The colored region boundaries are the $\mu \pm \sigma$ values for seven different measurements where the main cause of variation is power and alignment drift of the source to the spectrometer. The absorption spectrum of PDMS obtained in this manner corresponds well to the OSA benchmark measurement, see Fig. 5(d). A slight discrepancy for the longer wavelength channels is observed as expected. This is due to the fact that the central wavelength of the bandpass filter is not exactly centered to one FSR of the AWG, see Fig. 4(b). Taking the 
OSA spectrum as the true response of PDMS in this setup, calculations show that an idealized AWG with zero crosstalk and insertion loss would produce almost identical results. The idealized AWG is taken as an array of dirac functions at the AWG channel peak positions, see black crosses in Fig. 4(b). The Signal-to-Noise Ratio (SNR) for each channel of the system is found by comparing the signal strength at the modulation frequency in the reference measurement with the noise power in dark conditions at $\mathrm{f}_{\text {mod }}$, see Fig.5(b). The SNR is between $24-28 \mathrm{~dB}$ for 1 s integration time per channel. Using the input optical power spectrum from Fig.4(b) and the SNR for each channel, it is estimated that the lowest power change $\Delta P$ that can be detected (with $\mathrm{SNR}=1$ ) is between $-57.2 \mathrm{dBm} / \mathrm{nm}$ and $-69.2 \mathrm{dBm} / \mathrm{nm}$. For a given spectroscopic application where one would want to detect a minimum transmission change $\Delta \mathrm{T}$ of $0.1 \%(-30 \mathrm{~dB})$ with $\mathrm{SNR}=1$, a broadband source with a minimum power density of -27.2 $\mathrm{dBm} / \mathrm{nm}$ or a total power of $-10 \mathrm{dBm}$ within one FSR (50 $\mathrm{nm})$ is required. Although these values are adequate for most applications, there are several ways to further improve the performance of the device.

The detectivity of the photodetector can be increased by active cooling or by using an optical immersion microlens [35]. The input grating coupler can be replaced with a spot-size converter for edge-coupling for higher efficiency and larger optical bandwidth. Moreover, the input fiber should be fixed to avoid drift of the coupling efficiency to the spectrometer during the measurement. The channels can be addressed simultaneously at a different modulation frequency instead of time-sequentially, thus increasing the measurement time per channel, thereby increasing SNR. For testing purposes, the output grating coupler array in this design is sized to match the mode field diameter of the single-mode fibers. However, the array can be readily shrunk ten-fold for better collection efficiency or to allow for more output channels.

Finally for applications where large environmental temperature variations are present, the central wavelength of the device will shift by $\approx 0.125 \mathrm{~nm} / \mathrm{K}$ at $2.3 \mu \mathrm{m}$ wavelength, resulting in a small offset of the wavelength corresponding with each channel. To mitigate this, one can either use a temperature controller underneath the photonic chip, use specially designed athermal circuits [36] or use $\mathrm{SiN}$ waveguide circuits with a five times lower thermo-optic coefficient [37].

\section{Conclusions}

Low-cost and miniaturized spectrometers can be realized by combining an off-the-shelf mid-infrared photodetector with SOI wavelength demultiplexer circuits. A novel approach is shown where a single discrete photodetector is used to characterize the response of a twelve channel mid-infrared AWG at $2.3 \mu \mathrm{m}$. The different AWG channels are discerned by time-sequentially modulating the output arms with MZIbased thermo-optic modulators. The driving and read-out of the spectrometer is performed using low-cost standard electronic components. The absorption spectrum of PDMS is recovered at $2.3 \mu \mathrm{m}$ to good agreement with bench-top OSA measurements. This is a promising approach which can be applied to longer wavelength SOI spectrometers for the 3-4 $\mu \mathrm{m}$ wavelength range where high-performance cooled detectors are needed.

\section{ACKNOWLEDGMENT}

The authors thank Barry Smalbrugge from TU/e for wirebonding and CMST, Ghent University for providing the PDMS sample. 


\section{REFERENCES}

[1] R. Soref, "The past, present, and future of silicon photonics," IEEE $J$. Sel. Top. Quantum Electron., vol. 12, no. 6, pp. 1678-1687, Nov 2006.

[2] M. Pantouvaki et al., "Active components for $50 \mathrm{~Gb} / \mathrm{s}$ NRZ-OOK optical interconnects in a silicon photonics platform," J. Lightw. Technol., vol. 35, no. 4, pp. 631-638, Apr 2017.

[3] M. H. Khan et al., "Ultrabroad-bandwidth arbitrary radiofrequency waveform generation with a silicon photonic chip-based spectral shaper," Nat. Photonics, vol. 4, no. 2, p. 117, Feb 2010.

[4] A. Abbasi et al., "III-V-on-Silicon C-band high-speed electro-absorption modulated DFB laser," J. Lightw. Technol., Aug 2017.

[5] A. E.-J. Lim et al., "Review of silicon photonics foundry efforts," IEEE J. Sel. Top. Quantum Electron., vol. 20, no. 4, pp. 405-416, Jul 2014.

[6] J. T. Kindt, M. S. Luchansky, A. J. Qavi, S.-H. Lee, and R. C. Bailey, "Subpicogram per milliliter detection of interleukins using silicon photonic microring resonators and an enzymatic signal enhancement strategy," Anal. Chem., vol. 85, no. 22, pp. 10 653-10 657, Nov 2013.

[7] E. Ryckeboer, R. Bockstaele, M. Vanslembrouck, and R. Baets, "Glucose sensing by waveguide-based absorption spectroscopy on a silicon chip," Biomed. Opt. Express, vol. 5, no. 5, pp. 1636-1648, May 2014.

[8] Y. Chen, H. Lin, J. Hu, and M. Li, "Heterogeneously integrated silicon photonics for the mid-infrared and spectroscopic sensing," ACS Nano, vol. 8, no. 7, pp. 6955-6961, Jun 2014.

[9] Y. Li, P. Segers, J. Dirckx, and R. Baets, "On-chip laser Doppler vibrometer for arterial pulse wave velocity measurement," Biomed. Opt. Express, vol. 4, no. 7, pp. 1229-1235, Jul 2013.

[10] H. Yan et al., "Specific detection of antibiotics by silicon-on-chip photonic crystal biosensor arrays," IEEE Sens. J., vol. 17, no. 18, pp. 5915-5919, Sep 2017.

[11] Y. E. Marin, T. Nannipieri, C. J. Oton, and F. Di Pasquale, "Integrated FBG sensors interrogation using active phase demodulation on a silicon photonic platform," J. Lightw. Technol., vol. 35, no. 16, pp. 3374-3379, Aug 2017.

[12] A. Trita et al., "Simultaneous interrogation of multiple fiber bragg grating sensors using an arrayed waveguide grating filter fabricated in SOI platform," IEEE Photon. J., vol. 7, no. 6, pp. 1-11, Dec 2015.

[13] T. H. Stievater et al., "Trace gas absorption spectroscopy using functionalized microring resonators," Opt. Lett., vol. 39, no. 4, pp. 969972, Feb 2014.

[14] N. A. Yebo et al., "Selective and reversible ammonia gas detection with nanoporous film functionalized silicon photonic micro-ring resonator," Opt. Express, vol. 20, no. 11, pp. 11 855-11 862, May 2012.

[15] P. Larkin, Infrared and Raman Spectroscopy; Principles and Spectral Interpretation. Elsevier, 2011.

[16] R. Soref, "Mid-infrared photonics in silicon and germanium," Nat. Photonics, vol. 4, no. 8, pp. 495-497, Aug 2010.

[17] G. Roelkens et al., "Silicon-based heterogeneous photonic integrated circuits for the mid-infrared," Opt. Mater. Express, vol. 3, no. 9, pp. 1523-1536, Sep 2013.

[18] G. Z. Mashanovich et al., "Silicon photonic waveguides and devices for near-and mid-IR applications," IEEE J. Sel. Top. Quantum Electron., vol. 21, no. 4, pp. 407-418, Jul 2015.

[19] R. Wang et al., "III-V-on-silicon photonic integrated circuits for spectroscopic sensing in the $2-4 \mu \mathrm{m}$ wavelength range," Sensors, vol. 17, no. 8, p. 1788, Aug 2017.

[20] J. S. Penadés et al., "Suspended silicon mid-infrared waveguide devices with subwavelength grating metamaterial cladding," Opt. Express, vol. 24, no. 20, pp. 22908-22916, Oct 2016.

[21] S. A. o. Miller, "Low-loss silicon platform for broadband mid-infrared photonics," Optica, vol. 4, no. 7, pp. 707-712, Jul 2017.

[22] B. G. Lee et al., "DFB quantum cascade laser arrays," IEEE J. Quantum Elec., vol. 45, no. 5, pp. 554-565, May 2009.

[23] A. Spott et al., "Quantum cascade laser on silicon," Optica, vol. 3, no. 5, pp. 545-551, May 2016.

[24] L. Bizet et al., "Multi-gas sensing with quantum cascade laser array in the mid-infrared region," Appl. Phys. B, vol. 123, no. 5, p. 145, May 2017.

[25] R. Wang et al., "Broad wavelength coverage $2.3 \mu \mathrm{m} \mathrm{III-V-on-silicon}$ DFB laser array," Optica, vol. 4, no. 8, pp. 972-975, Aug 2017.

[26] P. Barritault et al., "Design, fabrication and characterization of an AWG at $4.5 \mu \mathrm{m}$," Opt. Express, vol. 23, no. 20, pp. 26 168-26181, Oct 2015.

[27] A. Vasiliev, M. Muneeb, R. Baets, and G. Roelkens, "High resolution silicon-on-insulator mid-infrared spectrometers operating at $3.3 \mu \mathrm{m}$," in Photon. Soc. Summ. Top. Meet. Ser. (SUM). IEEE, Jul 2017, pp. 177178.
[28] M. Nedeljkovic et al., "Mid-infrared silicon-on-insulator Fouriertransform spectrometer chip," IEEE Photon. Technol. Lett., vol. 28, no. 4, pp. 528-531, Feb 2016

[29] M. Erfan et al., "On-chip micro-electro-mechanical system fourier transform infrared (MEMS FT-IR) spectrometer-based gas sensing," Appl. Spectr., vol. 70, no. 5, pp. 897-904, May 2016.

[30] X. Nie, E. Ryckeboer, G. Roelkens, and R. Baets, "CMOS-compatible broadband co-propagative stationary Fourier transform spectrometer integrated on a silicon nitride photonics platform," Opt. Express, vol. 25, no. 8, pp. A409-A418, Apr 2017.

[31] M. Muneeb et al., "III-V-on-silicon integrated micro - spectrometer for the $3 \mu \mathrm{m}$ wavelength range," Opt. Express, vol. 24, no. 9, pp. 94659472, May 2016.

[32] P. Munoz, D. Pastor, and J. Capmany, "Modeling and design of arrayed waveguide gratings," J. Lightw. Technol., vol. 20, no. 4, p. 661, Apr 2002.

[33] R. Wang et al., "III-V-on-silicon 2- $\mu$ m-wavelength-range wavelength demultiplexers with heterogeneously integrated InP-based type-II photodetectors."

[34] R. Baets, D. Delbeke, G. Roelkens, and W. Bogaerts, "Integrated spectrometers with single pixel detector," Mar. 23 2017, US Patent App. $15 / 305,713$

[35] J. Piotrowski, W. Galus, and M. Grudzien, "Near room-temperature ir photo-detectors," Infrared Phys., vol. 31, no. 1, pp. 1-48, 1991.

[36] L. Wang et al., "Athermal arrayed waveguide gratings in siliconon-insulator by overlaying a polymer cladding on narrowed arrayed waveguides," Appl. Opt., vol. 51, no. 9, pp. 1251-1256, 2012.

[37] P. T. Lin et al., "Low-stress silicon nitride platform for mid-infrared broadband and monolithically integrated microphotonics," Adv. Opt. Mat., vol. 1, no. 10, pp. 732-739, 2013. 


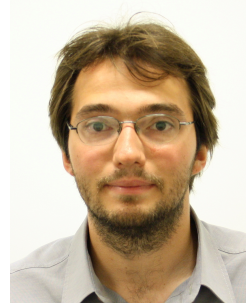

the OSA Optical Society.

Anton Vasiliev received a B.Sc. degree in applied physics engineering in 2012 and the M.Sc. degree in photonics in 2014 from Ghent University, Ghent, Belgium. He is currently working toward the Ph.D. degree in photonics with the Photonics Research Group, Department of Information Technology, imec, Ghent University, Ghent, Belgium. His current research interests include integrated circuits on SOI platform for mid-infrared spectroscopy applications and photothermal methods for trace gas detection. $\mathrm{He}$ is a member of the IEEE Photonics Society and

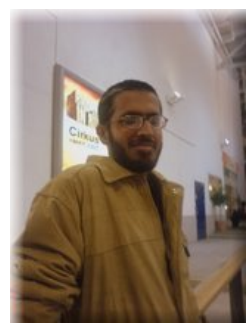

Muhammad Muneeb was born in 1982 in Pakistan. He received the B.Sc. degree in electronic engineering from the GIK Institute of Engineering and Technology, Swabi Khabar Pakhtoon Khwa, Pakistan, in 2005, and the Erasmus Mundus Masters degree in photonics from Royal Institute of Technology, Stockholm, Sweden and Ghent University, Ghent, Belgium, in 2010. Since November 2010, he is currently working in the Photonics Research Group, Ghent University where his research focus is on IIIV/Si photonic circuits for mid-infrared spectroscopic applications.

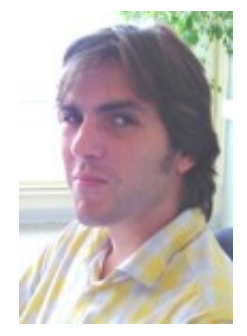

Jeroen Allaert received M.Sc. degree in electronics with specialization in design techniques in 2006 from University College Ghent (HoGent), Ghent, Belgium. He was with the Photonics Research Group, Ghent University, Ghent, Belgium from 2006 till 2017. He founded and is currently with Analogue Renaissance, a company specialized in electronics for musical purposes and custom design, which was incorporated as Cask Strength Electronics in 2016.

Joris Van Campenhout received the Masters degree in physics engineering, in 2002, and the Ph.D. degree in electrical engineering, in 2007, both from Ghent University, Ghent, Belgium. After his Ph.D., he worked as a Postdoctoral researcher at the IBM TJ Watson Research Center in New York, NY, USA. In 2010, he started at IMEC, Belgium, where he currently is the Program Manager Optical I/O.

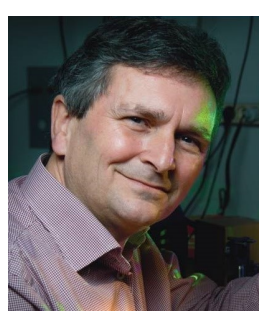

Roel Baets is full professor at Ghent University (UGent) and is associated with IMEC. He received an MSc degree in Electrical Engineering from UGent in 1980 and a second MSc degree from Stanford in 1981. He received a $\mathrm{PhD}$ degree from UGent in 1984. From 1984 till 1989 he held a postdoctoral position at IMEC. Since 1989 he has been a professor in the Faculty of Engineering and Architecture of UGent where he founded the Photonics Research Group. From 1990 till 1994 he has also been a part-time professor at Delft University of Technology and from 2004 till 2008 at Eindhoven University of Technology. He has mainly worked in the field of integrated photonics. As part of a team of 8 professors Roel Baets leads the Photonics Research Group. In 2006 he founded ePIXfab, the globally first Multi-Project-Wafer service for silicon photonics. Since then ePIXfab has evolved to become the European Silicon Photonics Alliance. He is also director of the multidisciplinary Center for Nano- and Biophotonics (NB Photonics) at UGent, founded in 2010. He was co-founder of the European MSc programme in Photonics. Roel Baets is an ERC grantee of the European Research Councile and a Methusalem grantee of the Flemish government. He is a Fellow of the IEEE, of the European Optical Society (EOS) and of the Optical Society (OSA). He is also a member of the Royal Flemish Academy of Belgium for Sciences and the Arts.

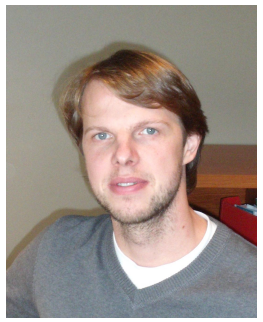

Günther Roelkens received the degree in electrical engineering in 2002 and the Ph.D. degree both from Ghent University, Ghent, Belgium, in 2007, from the Department of Information Technology (INTEC), where he is currently an Associate Professor. In 2008, he was a Visiting Scientist in the IBM T. J. Watson Research Center, Rochester, NY, USA. He is an Assistant Professor at Eindhoven University of Technology, Eindhoven, The Netherlands. His research interests include the heterogeneous integration of III-V semiconductors and other materials on top of silicon waveguide circuits and electronic/photonic cointegration. He was holder of an ERC starting grant (MIRACLE), to start up research in the field of integrated mid-infrared photonic integrated circuits. 